\title{
Probability of signaled reinforcement in multiple variable-interval schedules*
}

\author{
RONALD G. HUGHES \\ University of North Carolina at Greensboro, Greensboro, N.C. 27412
}

After responding was maintained on multiple variable-interval schedules of reinforcement, a signaling procedure was added to one component. The signaling procedure consisted of illuminating the key, the only source of illumination in the chamber, only when responding would be reinforced (probability of signaled reinforcement equals 1.00). Rate of responding in the unchanged component increased. When an equal number of "unreinforced" signals were added to the existing schedule (probability of signaled reinforcement equals .50), responding in the unchanged component increased further for two birds. Obtained rates of reinforcement were equal throughout the experiment.

In two-component multiple variable-interval schedules of reinforcement, responding in one component is affected by changes in reinforcement rate in a second component (Reynolds, 1961; Lander \& Irwin, 1968; Hughes, 1970). The inverse relation between responding in one component of multiple variable-interval schedules and reinforcement rate in a second component was well substantiated in a series of experiments by Hughes (1970), in which responding in one component was effectively suppressed by use of a signaled reinforcement procedure. Three-minute components of red alternated with 3-min components of blue illumination of the response key. When reinforcements were signaled in the red component, the key was illuminated only when a reinforcement was programmed. During the remainder of the red component, the key was not illuminated and the chamber was dark. No change in illumination occurred in the regularly alternated blue component. When illumination of the key in the signaled component was correlated with the availability of food on $100 \%$ of the occasions of its occurrence, responding in the unchanged VI component was a decreasing negatively accelerated function of the rate of reinforcement in the signaled component. The major significance of these data is that the distribution of obtained points along this function was similar to that obtained when reinforcement rate is varied with nonsignaled VI procedures; more importantly, this same interaction will occur in the absence of large variations in rate of responding in the component in which reinforcement rate is varied. However, many parameters of this procedure have yet to be investigated. For instance, by increasing the number of signal presentations one can directly manipulate response rate in the signaied component. The limiting condition of such a procedure would approach continuous illumination of the key. In the present experiment, additional "unreinforced" signal presentations were added to the schedule of signaled food presentations already in effect in order to assess the effects on responding in an unchanged VI component. The present design, in addition, made possible a direct replication of the earlier Brownstein \& Hughes (1970) results.

\section{SUBJECTS}

Three adult White Carneaux pigeons, all with previous experimental histories, were maintained at approximately $80 \%$ of their free-feeding weights. All animals were housed in individual cages with free access to water and with continuous artificial illumination. Daily experimental sessions were run.

\section{APPARATUS}

A standard two-key experimental chamber for pigeons similar in design to that described by Ferster \& Skinner (1957) was used. The right key was covered throughout the experiment. The left key could be transilluminated with either red or green light and was effectively operated by a force of $15 \mathrm{~g}$. No feedback relay was used, nor was any houselight present. White noise masked extraneous sounds. Reinforcement consisted of a 4.5-sec access to mixed grain. Reinforcements were programmed by variable-interval tapes consisting of an arithmetic series of 13 intervals arranged in an irregular order. Programming of the percentage condition was accomplished by use of a stepper programmed to present randomly half of the intervals on an arithmetic VI 1-min tape as "unreinforced" signals and half as "reinforced" signals. Standard relay programming equipment was employed and located in an adjoining room.

$$
\text { PROCEDURE }
$$

Three pigeons were exposed initially to multiple variable-interval schedules of reinforcement in which 3-min components of red regularly alternated with 3 -min components of green illumination of the response key. Responding was reinforced in each component according to identical arithmetic VI 2-min schedules, a daily session consisting of 10 presentations of each schedule component. When responding was approximately equal in both components, each bird was exposed to 10 consecutive daily sessions in which reinforcements were signaled in the red component. When reinforcements were signaled in the red component, the key was illuminated only when a reinforcement was programmed. During the remainder of the red component, the key was not illuminated and the chamber was dark. No change in illumination occurred in the regularly alternated green component. During these sessions, the probability of signaled reinforcement was 1.00 . No change in reinforcement rates occurred during these changes. For the 10 daily sessions which followed, the probability of signaled reinforcement was .50. This was accomplished without changing the rate of food reinforcement by programming additional "unreinforced" signals. Both reinforced and unreinforced signals were programmed by the same VI 1 -min tape. Unreinforced signal responses simply extinguished the key light and started the tape running again. No other programmed stimuli followed unreinforced signal presentations. Following 10 sessions in which the probability of signaled reinforcement was $.50,10$ sessions were run under the original signaled condition (probability of signaled reinforcement equaling 1.00). Throughout the experiment, the time from the onset of the signal to the first response, either reinforced or unreinforced, was recorded.

\section{RESULTS}

The primary observation was the rate of responding in the green, unchanged VI component. Panel A of Fig. 1 shows the response rates for individual animals in each component of the multiple schedule prior to the introduction of the signaled reinforcement condition. Panel B of Fig. 1 shows the response rate in the unchanged VI component when reinforcements were signaled in the red VI component. Throughout Panel $B$ the probability of signaled reinforcement was 1.00 . The effect of introducing the signaling procedure was to produce an immediate reduction in responding in the component in which reinforcements were signaled. None of the birds responded during the periods of blackout occurring between signal presentations, and all responded immediately to the signaled key whenever 


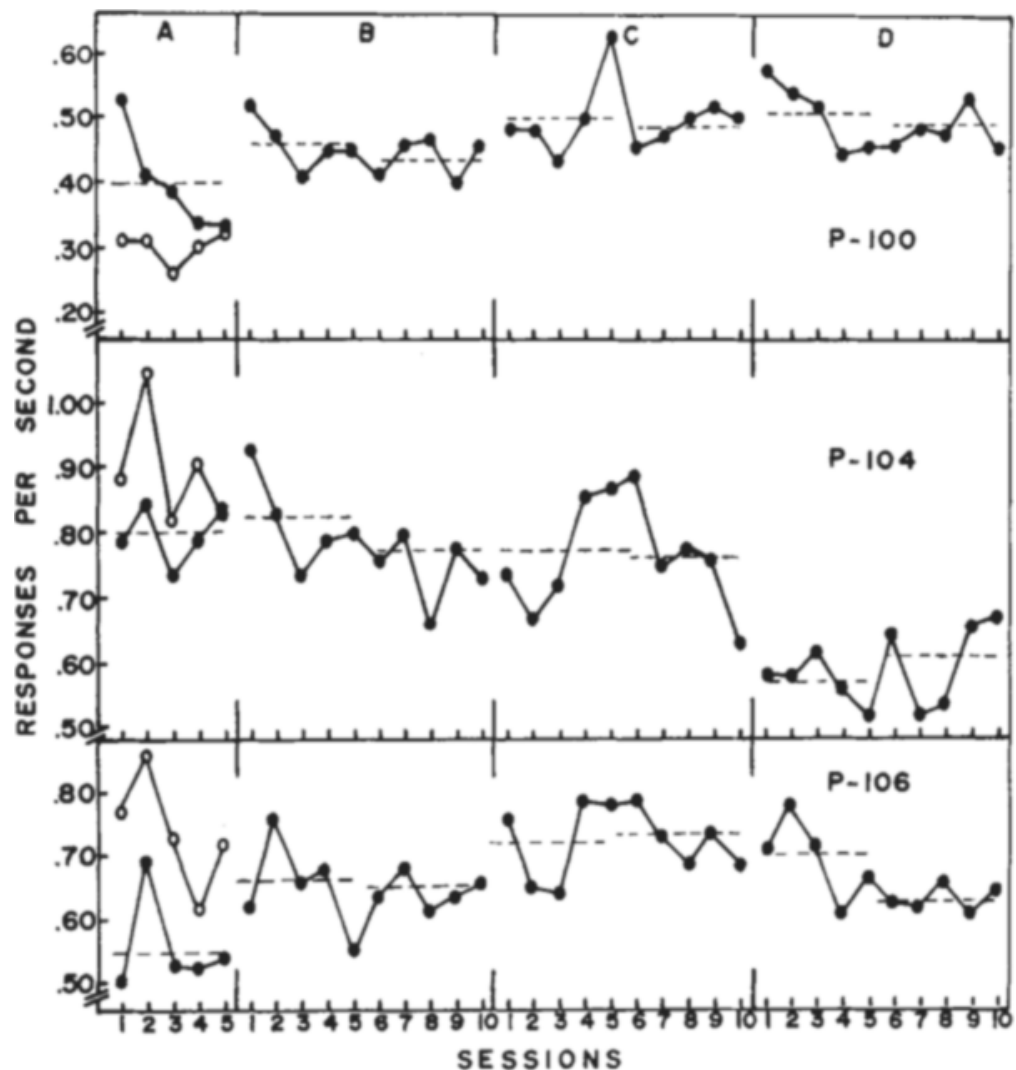

Fig. 1. Absolute response rates per session in unsignaled VI component (closed circles). See text for details.

it was illuminated. The effect observed in the unchanged VI component was an immediate increase in responding apparent on Session 1 following the change to signaled reinforcement. For all three animals, the mean response rate during the first 5 days of signaled reinforcement was higher than the mean response rate for the 5 days preceding the introduction of the signal. This increase in responding was sustained for Pigeons 100 and 106, but for Pigeon 104 responding by the third session had decreased below the previous unsignaled level and remained at or below this level for the remainder of the 10 sessions. Panel $\mathrm{C}$ shows the response rates in the unsignaled VI component when additional unreinforced signals were added to the existing VI 2-min schedule of food-reinforced signals already in effect. Reinforcements per session in each component remained unchanged by the addition of the unreinforced signals. Changing the probability of signaled reinforcement from 1.00 to .50 produced a further increase in responding in the unchanged component for two birds. Mean response rates for the first 5 days for Pigeons 100 and 106 increased above those maintained by the previous signaled condition. Again little change in mean response rate occurred from the first to the last 5 days under this condition. All birds tended to show peaks in responding occurring between Session 4 and Session 6. Panel D shows response rates in the unchanged component when the original signal conditions were reinstated. An increase in responding occurred for Pigeons 100 and 106 for Sessions 1-3 when compared to the rate on the last day of the preceding percentage condition. Little change was seen in the mean response rates for Pigeon 100, although a decrease in responding did occur over Sessions 4-6. Pigeon 106 showed an almost complete recovery of its earlier performance. The decrease in response rate that occurred for Pjgeon 104 is difficult to interpret as to whether it represents the same effect as was clearly observed in Pigeon 106, and to a lesser extent in Pigeon 100, or whether the decrease was simply a continuation of a downward trend in responding begun during the preceding experimental condition. In fact, the results for Pigeon 104 seem to show a general decreasing trend across all conditions and are therefore to be viewed with caution. Two of the birds, however, were consistent in showing an initial increase upon introduction of the signal and a further increase when additional unreinforced signals were added to the variable-interval schedule of food reinforcement which was in effect throughout. Furthermore, the time between the onset of the signal and the first response following the onset remained almost constant across conditions.

\section{DISCUSSION}

The results of the present experiment clearly substantiate the finding reported by Brownstein \& Hughes (1970). Although the increases in responding in the unchanged VI component were smaller in the present instance, these differences are possibly accounted for by the fact that Brownstein and Hughes used naive birds while the present study used birds with extensive prior histories. Too, it is sometimes observed that responding on VI schedules for long periods of time may render a pigeon's responding insensitive to changes in the experimental variables, i.e., a locked rate may develop (see Sidman, 1960, 176-177).

The most interesting finding is the increase in responding which occurred in the unchanged $\mathrm{VI}$ component when unreinforced signals were added to the variable-interval schedule of food already in effect. The results of the present experiment can be related to three areas: (1) frustrative nonreward (Amsel, 1958), (2) reinforcement omission (Staddon \& Innis, 1966), and (3) conditioned reinforcement (Kelleher \& Gollub, 1962). Procedural similarities exist between the multiple-schedule situation in operant-conditioning studjes and the double-runway studies of Amsel. Following reinforcement in two successive components or runways, some parameter of reinforcement such as rate or magnitude is then decreased. In both instances, responding is observed to increase in the unchanged schedule component or runway. These results have been well documented in the operant-conditioning literature under the headings of behavioral contrast and multiple-schedule interactions, and only recently (Bloomfield, 1967, 1969) has serious appeal been made to the use of frustration as an explanatory tool in interpreting these data.

The reinforcement-omission studies of Staddon lend themselves to conceptualization in terms of multiple-schedule interactions in that response rate in an unchanged component is higher when reinforcement rate is decreased in a second component. Staddon's data deal, however, with responding maintained by fixed-interval (FI) schedules of reinforcement and are plausibly accounted for on the basis of the discriminative control exerted over the postreinforcement pause by the occasion of reinforcement. The interpretation placed on Staddon's data is not directly applicable in the present situation involving rate changes under $\mathrm{VI}$ schedules of 
reintorcement. Additionally, the runway studies and the reinforcement-omission studies differ from the present study in that reinforcement frequency (as measured by number of reinforcements per session in each schedule component) underwent a change. No change in reinforcements per session occurred in the present experiment.

It should be noted that the procedure used is identical in many respects to procedures used to establish a stimulus as a conditioned reinforcer (Kelleher \& Gollub, 1962). Following Schuster's (1969) assumption, when two identical food schedules are in effect, the addition of a schedule of conditioned reinforcers conjointly to one schedule should increase the "reinforcement value" of responding on that schedule and consequently produce a rate difference between the two food schedules. Although Schuster's failure to find a contrast effect is perhaps explained by the rate requirement in effect for the schedule of conditioned reinforcement, the increase in response rates in the present experiment do not seem to be predictable on the basis of conditioned-reinforcement theory alone. Instead. the "reinforcing vafue" of the schedule in the signaled component seems to have been diminished, rather than enhanced, by the addition of the unreinforced signals.

The effect produced by reducing the probability of signaled reinforcement in this way seems to resemble more the effect obtained by Brethower \& Reynolds (1962) when a mild electric shock was added to one component. The results of their experiment along with the present results seem to be in line with Bloomfield's (1969) assertion that the occurrence of contrast in multiple schedules is in some sense a result of a "change for the worse" in one component. Among the class of events suggested by Bloomfield as a "worsening of conditions" are reduction in reinforcement frequency, the introduction of punishment, and restraint involved in the development of inhibition. Interpreted in these terms, the present results simply specify an additional condition in which an increase in responding occurs in a schedule component where such an increase is "uncalled for" by the contingencies.

A suggested alternative explanation for these data is the following: To the extent that one may still think of signaled $\mathrm{VI}$ as maintaining the essential characteristics of a variable-interval schedule of reinforcement, it would seem equally correct to think of the unreinforced signal presentations as a schedule of signaled extinction. Thus, it is possible to consider the condition in which the probability of signaled reinforcement is .50 as a mixed schedule composed of signaled VI and signaled extinction. Considered in this

way, the transition in the signaled component from signaled VI 2 min to $m i x$ (signaled VI 2 min. signaled extinction) represents a decrease in the reinforcement "value" of responding in the signaled component. That animals prefer multiple to mixed schedules of reinforcement, when both provide the same rate of food reinforcement, is well documented (Bower, McLean, \& Meacham, 1966) and lends support to the present interpretation of these results. Experiments are currently under way to determine if a gradation of these effects occurs over a wider range of probability values. The procedure is clearly a useful and promising one for investigating topics in each of the areas discussed.

\section{REFERENCES}

AMSEL, A. The role of frustrative nonreward in noncontinuous reward situations. Psychological Bulletin, 1958, 55, 102-119.

BLOOMFIELD. T. M. Frustration, preference, and behavioural contrast. Quarterly Journal of Experimental Psychology, 1967, 19, 166-169. BLOOMFIELD, T. M. Behavioural contrast and the peak shift. In R. M. Gilbert and N. S. Sutherland (Eds.), Animal discrimination learning. New York: Academic Press, 1969. Pp. 000-000.

BOWER, G., McLEAN, J., \& MEACHAM, J. Value of knowing when reinforcement is due. Journal of Comparative \& Physiological Psychology, 1966, 62, 184-192.

BRETHOWER, D. M., \& REYNOLDS, G. S. A facilitative effect of punishment on unpunished behavior. Journal of the

\section{Avoidance of thermal stimuli in the rat}

\section{T. JAMES MATTHEWS*, LAWRENCE P. MORIN + , and RUSSELL M. CHURCH Brown University, Providence, R.I. 02912}

The study of thermally motivated instrumental behavior was extended to the avoidance-conditioning paradigm. Six Ss were trained on a thermal Sidman avoidance schedule. The UCS temperature and the ambient temperature were varied, while the temporal properties of the schedule were held constant. The results indicated that whenever the difference between the two thermal conditions is sufficient to sustain responding, the probability of an avoidance response is related to the mean of the UCS and ambient temperatures.

In the investigation of the thermally motivated instrumental behavior of the rat, there has been a consistent concern with an instrumental response that immediately improves the thermal condition of the animal (Carlton \& Marks, 1958; Weiss \& Laties, 1960: Carlisle, 1969). The usual procedure requires the $S$ to press a bar to escape from a stressful drive condition into a stress-reducing reinforcement condition. This procedure has been accepted as the elementary paradigm of behavioral thermoregulation. Another way in which animals may thermoregulate is by avoiding

*Please send requests for reprints to the senior author at the Department of Psychology. New York University. University Heights. New York. New York 10453

$\div$ Now at Rutgers University, Newark, New Jersey 07102. stressful conditions. The present study is designed to study thermally motivated avoidance behavior.

There are two important differences between the avoidance and escape procedures. In the avoidance procedure, the instrumental response occurs in the presence of the less severe of the two thermal conditions, and does not produce an immediate change in temperature.

In the escape procedure the influence of the more-severe thermal condition (drive) and the less-severe thermal condition (reinforcer) have been given thorough parametric analysis (Matthews, in press) The present study provides an analysis of the comparable parameters in the avoidance paradigm. Those variables are the temperature of the more-severe stimulus (UCS) and of the less-severe 